\title{
Eficacia de una combinación analgésica en perras sometidas a ovariohisterectomía
}

\author{
López, J.E.; Guaimas Moya, L.E.; Baez, A.D.; Lockett, M.B.; Repetto, C.J.
}

Hospital de Clínicas, Cátedra de Cirugía y Anestesiología y Cátedra de Clínica de Pequeños Animales, Facultad de Ciencias Veterinarias, Universidad Nacional del Nordeste (UNNE), Sargento Cabral 2139,

Corrientes (3400), Argentina. Tel/fax 03783-425753 E-mail: tecquir@vet.unne.edu.ar.

\begin{abstract}
Resumen
López, J.E.; Guaimas Moya, L.E.; Baez, A.D.; Lockett, M.B.; Repetto, C.J.: Eficacia de una combinación analgésica en perras sometidas a ovariohisterectomía. Rev. vet. 20: 2, 116-120, 2009. El objetivo del trabajo fue evaluar los efectos de una combinación analgésica conformada por dextropropoxifeno, lidocaína, xilacina y ketamina en seis perras sometidas a ovariohisterectomía. La premedicación se realizó con dextropropoxifeno y xilacina, en tanto que la inducción se llevó a cabo con propofol y diazepam. La perfusión de la combinación analgésica ensayada comenzó luego de la intubación endotraqueal, mediante la cual un vaporizador de isoflurano suministró una concentración inicial de 3\%, la cual fue variando según los requerimientos del animal (1,5-3\%). El oxígeno se aportó a razón de $20 \mathrm{ml} / \mathrm{kg} /$ min. No se registraron modificaciones importantes de parámetros como frecuencia cardíaca, temperatura rectal y electrocardiografía, los cuales fueron evaluados en ocho tiempos distintos, que se iniciaron 5 minutos antes de la premedicación anestésica. Hacia la mitad de la experiencia, la frecuencia respiratoria se registró en los límites inferiores. La presión arterial acusó elevaciones iniciales coincidentes con los momentos de mayor estimulación nociceptiva. Como conclusión preliminar surge que la combinación ensayada produjo una adecuada analgesia sin causar alteraciones de la estabilidad cardiorrespiratoria.
\end{abstract}

Palabras clave: perro, cirugía, analgesia, dextropropoxifeno, lidocaína, xilacina, ketamina.

\begin{abstract}
López, J.E.; Guaimas Moya, L.E.; Baez, A.D.; Lockett, M.B.; Repetto, C.J.: Efficacy of an analgesic combination in canine ovariohysterectomy. Rev. vet. 20: 2, 116-120, 2009. The objective of this work was to evaluate the effects of an analgesic combination consisted of dextropropoxifene, lidocaine, xylazine and ketamine in six bitches submitted to ovariohysterectomy. The premedication was made with dextropropoxifene and xylazine, whereas the induction was carried out with propofol and diazepam. The perfusion of the analgesic combination was initiated after endotraqueal intubation, using an isoflurane vaporizer which provided an initial concentration of 3\%, varying from 1.5 to $3 \%$ according to anesthesia requirements. Oxygen was given at the rate of $20 \mathrm{ml} / \mathrm{kg} / \mathrm{min}$. Modifications of parameters such as cardiac frequency, rectal temperature and electrocardiography were not registered, which were evaluated at different times, initiating 5 minutes before premedication. Towards half of the experiment, the respiratory frequency was in the inferior limits. The arterial pressure accused coincident initial elevations with the moments of greater nociceptive stimulation. As preliminary conclusion arises that the essayed combination produced a suitable analgesia without alterations of the cardiorespiratory stability.
\end{abstract}

Key words: bitch, ovariohysterectomy, analgesia, dextropropoxifene, lidocaine, xylazine, ketamine.

\section{INTRODUCCIÓN}

La analgesia perioperatoria debe ser considerada primordial porque de ella dependerá el éxito de la cirugía, a la par de una técnica quirúrgica depurada. Los mecanismos nerviosos que participan en las diferentes etapas de la respuesta a estímulos dolorosos son esen-

Recibido: 8 octubre 2009 / Aceptado: 9 noviembre 2009 cialmente similares en los animales y en el hombre. Los neurotransmisores, así como las vías nerviosas sobre las que actúan, operan según los mismos mecanismos de funcionamiento. Además, los umbrales necesarios para desencadenar la respuesta, muestran tantas diferencias entre seres humanos y animales como las diferencias que se observan entre distintas personas. Por ello se impone la necesidad de erradicar el concepto de que los animales son más tolerantes al dolor, ya que 
solo se diferencian de los seres humanos por la forma de expresarlo.

La tarea de impedir que el dolor se presente o permanezca se ha tornado más eficiente debido a los constantes avances del conocimiento en el área de la algiología, el mayor compromiso del médico veterinario con el bienestar de su paciente y desde luego, la expansión del arsenal terapéutico ${ }^{13}$.

La Sociedad Internacional para el Estudio del Dolor lo define como una experiencia sensoria y emocional desagradable, asociada con daño tisular real o potencial. Debido a inconvenientes en la medición del dolor animal, las instituciones pautaron que si un procedimiento quirúrgico ocasiona dolor en pacientes humanos adultos, los animales de laboratorio sometidos al mismo deben ser tratados con terapia analgésica ${ }^{7,8}$.

La evaluación del dolor se basa en la probabilidad, parámetros fisiológicos y respuestas conductuales. La probabilidad está influida por las experiencias clínicas. Los procedimientos con factibilidad de ser dolorosos incluyen amputaciones, toracotomías (en especial la esternotomía mediana), resecciones auriculares, ciertos procedimientos abdominales tales como la nefrectomía, prostatectomía y resección hepática, así como la reparación de fracturas pélvicas. La mayoría de los cambios fisiológicos comprenden la activación del sistema nervioso simpático. Los cambios en la frecuencia cardíaca, circulación periférica y patrones respiratorios pueden indicar dolor, angustia (aflicción) o malestar. Sin embargo, los parámetros fisiológicos no deben ser utilizados con exclusividad para evaluar el dolor, porque otras variables (administración de drogas, hipovolemia) pueden alterarlos.

El buen manejo del dolor post-operatorio comienza durante la etapa pre-operatoria. Las drogas sistémicas pueden ser administradas en forma programada y repetirlas las veces que sea necesaria dependiendo de la droga analgésica utilizada, en infusión continua o mediante absorción continua ${ }^{6}$.

El tramadol es un analgésico de acción central que pertenece a la "nueva clase de analgésicos". Es un compuesto sintético análogo de la codeína ${ }^{5}$, pudiendo ser utilizado como premedicación anestésica con el fin de mejorar la analgesia intraoperatoria de los anestésicos inhalados y proporcionar al paciente una recuperación confortable. La duración de su acción es de 6 a 8 horas, aportando un efecto analgésico residual durante las primeras horas del período de recuperación ${ }^{13}$.

Para la premedicación también se puede utilizar la xilacina, derivado tiazínico con propiedades sedantes analgésicas y relajantes musculares. Las dosis aconsejadas oscilan entre $0,4-1 \mathrm{mg} / \mathrm{kg}{ }^{13}$. La acción analgésica podría ser breve (15-30 minutos), mientras que la sedación persiste 1-2 horas. Los efectos cardiovasculares de esta droga son variables e incluyen elevación inicial de la tensión arterial, seguida de hipotensión. Parece sensibilizar al miocardio a las catecolaminas y acrecentar la probabilidad de disrritmias. Puede causar bradicardia, bloqueos sinoauricular y auriculoventricu- lar de primero y segundo grado, disociación auriculoventricular y arritmia sinusal acentuada, eventos que resultarían de la hiperactividad vagal y pueden contrarrestarse con atropina.

El propofol es un anestésico inyectable de acción ultracorta y de inicio rápido, formulado en una emulsión acuosa que contiene propofol $(10 \mathrm{mg} / \mathrm{ml})$, aceite de soja $(100 \mathrm{mg} / \mathrm{ml})$, glicerol $(2,5 \mathrm{mg} / \mathrm{ml})$, lecitina de huevo $(12 \mathrm{mg} / \mathrm{ml})$ e hidróxido de sodio para ajustar el $\mathrm{pH}$. Su rápida absorción en el $\mathrm{SNC}$ da lugar a un rápido inicio de la acción. Se puede usar para inducir y mantener la anestesia. La inducción de la anestesia es normalmente rápida y efectiva. La dosificación estimada para animales sanos no premedicados oscila entre $8-10 \mathrm{mg} /$ $\mathrm{kg}$, para premedicados entre 4 y $6 \mathrm{mg} / \mathrm{kg}^{7}$.

La ketamina es el disociativo más utilizado en anestesia de animales. Entre las respuestas características de la disociación se encuentra el desarrollo de un estado cataleptoide. Produce un estado de inconsciencia que depende de la dosis y de la analgesia. La analgesia producida por la ketamina tiene lugar a dosis subanestésica y los elevados umbrales del dolor se relacionan con concentraciones plasmáticas mayores o iguales a $0,1 \mathrm{mg} / \mathrm{ml}^{9}$.

El dextropropoxifeno es un analgésico químicamente relacionado con la metadona que no induce adicción. Su efecto analgésico es débil comparado con la codeína. Se utiliza en dolores moderados de índole visceral o muscular. Por lo general se combina con otros analgésicos con los que se acentúa su efecto ${ }^{14}$. El perfil hemodinámico del paciente se mantiene estable, aunque se registra una tendencia a la bradicardia. $\mathrm{La}$ combinación de dextropropoxifeno ( $2 \mathrm{mg} / \mathrm{kg}$ IV) con agonistas alfa-2 adrenérgicos incrementa tanto el efecto sedante como la eficacia analgésica, convirtiendo a la asociación en una excelente opción para el manejo del dolor agudo.

Para el mantenimiento de la anestesia se utilizan los anestésicos inhalatorios, éstos agentes son únicos porque se administran y se eliminan por vía pulmonar. Su extenso empleo en la anestesia de los animales se debe a sus características farmacocinéticas, que permiten controlar y modificar de forma rápida la profundidad anestésica ${ }^{3,4}$.

La potencia de los agentes anestésicos inhalatorios es expresada por su valor de concentración alveolar mínima (CAM) ${ }^{3}$, término que expresa la presión parcial del anestésico en el aire alveolar (medida en volúmenes por ciento) necesaria para que el $50 \%$ de los individuos no respondan con movimientos a un estímulo doloroso estandarizado. Para evitar que el otro $50 \%$ se mueva durante la cirugía, se eleva la dosis del anestésico hasta un punto en el que el $99 \%$ de los pacientes esté incapacitado de responder con movimientos a los estímulos dolorosos; este nivel se encuentra 1,3 veces por encima de la CAM (CAM x 1,3) ${ }^{13}$.

Numerosos estudios en especies animales documentan que los opioides reducen la CAM de un anestésico inhalatorio necesaria para prevenir las respuestas a 
un estímulo doloroso ${ }^{12}$. Los agentes anestésicos inhalados más utilizados en la actualidad son el isoflurano y el sevoflurano ${ }^{10}$. El isoflurano tiene un valor de CAM de $1,28 \%$ en el perro, lo cual se asocia con una rápida inducción y menor tiempo de recuperación ${ }^{1,3}$.

La lidocaína es uno de los anestésicos locales de mayor aplicación; ejerce su acción en forma rápida e intensa. Su alta liposolubilidad le permite acceder al sitio de acción en todo tipo de fibra nerviosa, por lo que en general produce tanto bloqueos sensitivos como motores. La administración de esta droga por vía endovenosa en infusión continua permite, merced a su efecto

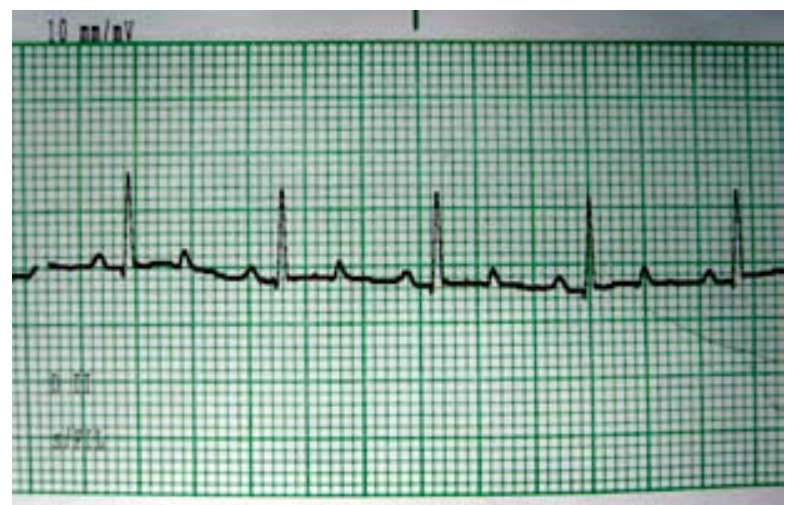

Figura 1. Electrocardiograma (D2, $25 \mathrm{~mm} / \mathrm{seg})$.

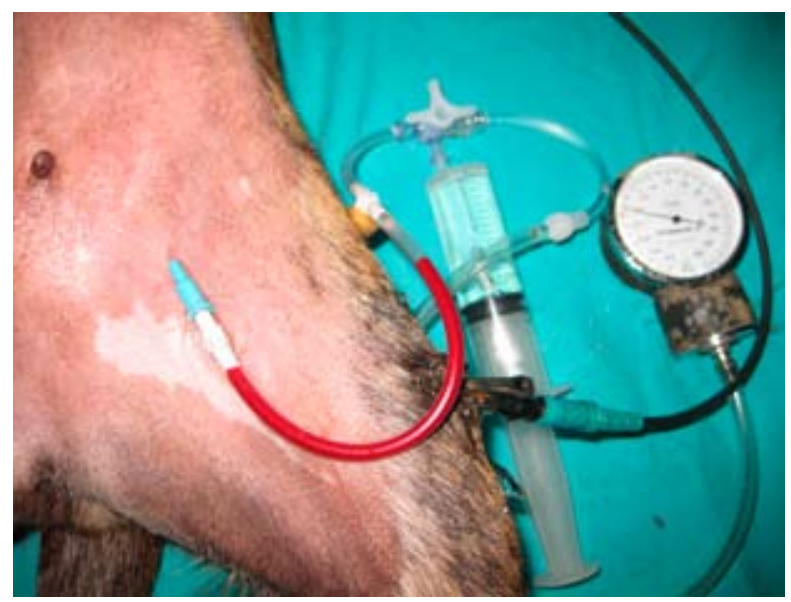

Figura 2. Determinación directa de presión arterial.

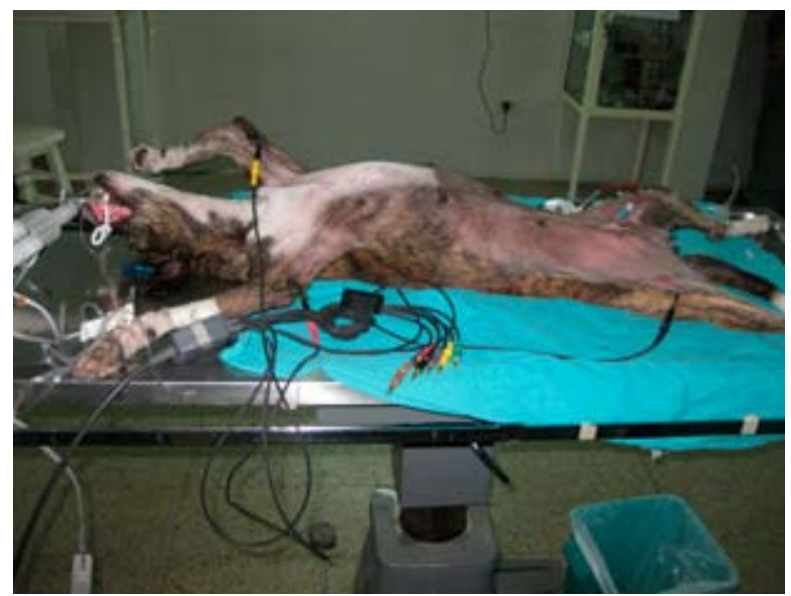

Figura 3. Mantenimiento de la anestesia y control electrocardiográfico. analgésico central, disminuir el aporte de anestésicos generales, reduciendo de manera significativa su dosis efectiva media (CAM). Además, es un excelente complemento cuando se agrega en combinación con otros analgésicos, como por ejemplo los opioides. En todos los casos, la infusión IV de anestésicos locales debe emplear soluciones libres de agentes vasoconstrictores, como la epinefrina ${ }^{13}$.

En cirugía abdominal, la administración IV de lidocaína al 2\% (bolo de $1,5 \mathrm{mg} / \mathrm{kg}$ en $10 \mathrm{~min}$, seguido de una infusión IV de $1,5 \mathrm{mg} / \mathrm{kg} / \mathrm{h}$ ), se ha asociado a una disminución de la intensidad del dolor al movimiento y del consumo de rescate opioide ${ }^{11}$.

Los objetivos del trabajo fueron determinar el efecto analgésico intraoperatorio de una combinación de drogas (ketamina, lidocaína, dextropropoxifeno y xilacina: KLDX) durante el periodo anestésico con isofluorano en perras sometidas a ovariohisterectomía. También se decidió constatar los efectos cardiorrespiratorios de KLDX durante el periodo anestésico, así como establecer el tiempo y la calidad de la recuperación en dichos animales.

\section{MATERIAL Y MÉTODOS}

La combinación analgésica KLDX se ensayó en seis perras clínicamente sanas, de diferentes razas, pesos y edades, sometidas a ovariohisterectomía con la finalidad de evitar la procreación. El compuesto se obtuvo diluyendo en solución fisiológica $1 \mathrm{mg} / \mathrm{kg}$ de dextropropoxifeno, $0,5 \mathrm{mg} / \mathrm{kg}$ de xilacina (dos tercios restantes de la dosis calculada para premedicación), 0,5 $\mathrm{mg} / \mathrm{kg}$ de lidocaína (sin epinefrina) y $1 \mathrm{mg} / \mathrm{kg}$ de ketamina, la cual se administró cada treinta minutos (dosis total $2 \mathrm{mg} / \mathrm{kg} /$ hora).

Antes de la premedicación se realizó un examen físico del paciente, determinándose las frecuencias cardíaca y respiratoria, temperatura rectal, tiempo de llenado capilar y electrocardiografía (Figura 1). Estas mediciones fueron realizadas cinco minutos antes de iniciar la premedicación anestésica (tiempo cero T0). Acto seguido se procedió a realizar la premedicación con dextropropoxifeno $1 \mathrm{mg} / \mathrm{kg}$ por vía subcutánea en la parrilla costal; treinta minutos después se aplicó xilacina en dosis de un tercio de $0,5 \mathrm{mg} / \mathrm{kg}$ por la misma vía.

Pasados diez minutos de esta última aplicación, se evaluaron nuevamente los parámetros antemencionados, así como la presión arterial directa (Figura 2). Este tiempo fue registrado en la ficha anestésica como tiempo 1 (T1). Inmediatamente se dio inicio a la inducción anestésica mediante la administración de propofol $4 \mathrm{mg} / \mathrm{kg}$ por vía EV más diazepam $0,2 \mathrm{mg} / \mathrm{kg}$ por la misma vía. Al mismo tiempo comenzó la perfusión continua de la combinación KLDX y posteriormente se efectuó la intubación endotraqueal.

A continuación los animales fueron conectados al equipo de anestesia inhalatoria con vaporizador de isoflurano, aportándose inicialmente una concentración de $3 \%$ (porcentaje de inducción), que se modificó a un 
Tabla 1. Modificaciones de las variables fisiológicas en los distintos tiempos.

\begin{tabular}{lcccccccc}
\hline parámetros & $\mathrm{T} 0$ & $\mathrm{~T} 1$ & $\mathrm{~T} 2$ & $\mathrm{~T} 3$ & $\mathrm{~T} 4$ & $\mathrm{~T} 5$ & $\mathrm{~T} 6$ & $\mathrm{~T} 7$ \\
\hline frecuencia cardiaca, rpm & 135 & 129 & 122 & 112 & 107 & 98.5 & 108 & 125 \\
frecuencia respirat., cpm & jadeo & 22 & 8 & 7 & 9 & 6 & 12 & 23 \\
temperatura, ${ }^{\circ}$ centigrados & 39,1 & 38,2 & 38,1 & 38,2 & 37,5 & 37,1 & 37,0 & 37,0 \\
presión arterial, mm Hg & & 100 & 120 & 117 & 128 & 120 & 100 & 80 \\
saturación de $\mathrm{O}_{2}, \%$ & & 95 & 95 & 98 & 96 & 96 & 98 & 98 \\
tiempo extubación, min & & & & & & & & $5^{\prime}$ \\
tiempo recuperación, min & & & & & & & $20^{\prime}$ \\
\hline
\end{tabular}

porcentaje de mantenimiento (1,5-3\%) según los requerimientos del animal (Figura 3). Además se suministró un flujo de oxígeno de $20 \mathrm{ml} / \mathrm{kg} /$ minuto. La saturación de oxígeno fue evaluada mediante oximetría de pulso.

Las variables fisiológicas fueron registradas en los siguientes tiempos quirúrgicos: antes de la incisión de piel y pared abdominal (T2), después de la incisión de piel y pared abdominal (T3), al ligar las arterias ováricas y uterinas izquierda y derecha (T4), durante la ligadura del cuello uterino (T5), al suturar piel y pared abdominal (T6) y quince minutos después de la suspensión de la administración de isoflurano (T7).

El vaporizador de isofluorano fue modificado para mantener el plano anestésico según las necesidades de los animales, las cuales se determinaron según los siguientes parámetros: (a) reflejo palpebral ausente (al estar presente se aumentó el aporte de isoflurano en $0,5 \%$ hasta la abolición de este reflejo); (b) reflejo retractor ocular presente débil y dilatación pupilar, estas dos variables fueron subjetivamente definidas por el evaluador para controlar la profundidad anestésica; (c) dolor profundo periostial falángico ausente, al estar presente en el inicio del período de mantención anestésica se aumentó en $0,5 \%$ esperando la desaparición de la respuesta al estímulo; (d) baja frecuencia respiratoria (menos de $8 / \mathrm{min}$ ): cuando disminuyó esta variable se redujo $0,5 \%$ el porcentaje de isofluorano (y así sucesivamente). Lo contrario se realizó cuando la frecuencia respiratoria aumentó por sobre los 20 ciclos por minuto; (e) elevación de la frecuencia cardiaca sobre $160 \mathrm{rpm}$, que se interpretó como una respuesta al dolor. En estos casos el vaporizador de isoflurano fué incrementado en $0,5 \%$ esperando respuesta, que se consideró positiva al reducirse la frecuencia cardíaca.

\section{RESULTADOS Y DISCUSIÓN}

La Tabla 1 muestra las variaciones cuantitativas de los parámetros fisiológicos en los distintos tiempos, destacándose que en ninguno de ellos, ni siquiera en T4 que es el momento de mayor estimulación nociceptiva, se produjeron frecuencias cardiacas que superen 160 rpm. Se advierte la disminución de la frecuencia respiratoria registrada desde $\mathrm{T} 2$ a T5, coincidente con los momentos de mayor estimulación nociceptiva, en los que se debió incrementar el porcentaje de isoflurano.

Cabe destacar que la concentración de lidocaina utilizada en perfusión no fue coincidente con las dosis utilizadas por otros autores ${ }^{2,13}$, a efectos de no acentuar la hipotensión que podría provocar esta droga combinada con otras como la xilacina, con similar efecto. La presión arterial media determinada directamente en la arteria femoral, sufrió leves variaciones en T3, T4 y T5. La respiración disminuyó a los límites más bajos en T3 y T6. Las variables cardiorrespiratorias cualitativas se mantuvieron normales; el ritmo cardíaco permaneció bajo control sinusal y la respiración fue de tipo toracoabdominal en todos los tiempos.

Si bien el número de animales experimentales es aún insuficiente para poder emitir conclusiones definitivas, resulta auspicioso que los datos obtenidos de las perras ovariohisterectomizadas revelan que la combinación KLDX generó respuestas adecuadas sin provocar alteraciones cardiorrespiratorias.

\section{REFERENCIAS}

1. Bau Marquez P. 2002. Farmacología del isoflurano. Rev Med Vet. Esp Peq Anim (Chile) 15: 66.

2. Bednarski RM. 2007. Dogs and cats. In: Lum \& Jones veterinary anesthesia and analgesia (Tranquilli WJ, Thurmon JC, Grimm KA, ed.), 4th ed., Blackwell Publishing, Ames (USA), p. 705-715.

3. Boothe MD. 2001. Small animal clinical farmacology and therapeutics, Saunders, Filadelfia, p. 339-346.

4. Botana LM, Landoni F, Martín-Jiménez T. 2002. Farmacología y terapéutica veterinaria, McGraw-Hill, Madrid, p. 125-126.

5. Dayer P, Desmeules J, Collart L. 1997 Pharmacology of tramadol. Drugs 53:18-24.

6. Fossum TW. 2004. Cirugía en pequeños animales, $2^{\circ} \mathrm{ed}$., Inter-Médica, Buenos Aires, p. 97-106.

7. Hardie E, Hansen B, Carol G. 1997 Behavior after ovariohysterectomy in the dog: what's normal. Appl Anim Behav Sci 51: 111-128.

8. Helyer PW, Robetson SA., Fails AD. 2007. Pain and its managements. In: Lum \& Jones veterinary anesthesia and analgesia (Tranquilli WJ, Thurmon JC, Grimm KA, ed.), 4th ed., Blackwell Publishing, Ames (USA), p. 31-52.

9. Hui Chu L. 2007. Dissociative anesthetics. In: Lum \& Jones veterinary anesthesia and analgesia (Tranquilli WJ, Thurmon JC, Grimm KA, ed.), 4th ed., Blackwell Publishing, Ames (USA), p. 301-305.

10. Ibancobichi JA. 2003. Examen preanestésico. Memorias VIII Curso Internacional MEVEPA (Tomé, Chile), On line: www.bibliodigital.udec.cl/sdx/UDEC2/tesis/pdf.

11. Koppert W, Weigand M, Neumann F. 2004. Perioperative intravenous lidocaine has preventive effects on postoperative pain and morphine consumption after major abdominal surgery. Anesth Analg 98: 1050-1055. 
12. Neira F, Ortega-García Jl. 2002. Nuevos opioides en el tratamiento del dolor crónico. Rev Soc Esp Dolor 9: 421422.

13. Otero PE. 2006. Dolor: evaluación y tratamiento en pequeños animales, Inter-Médica, Buenos Aires, p. 1-2, 100, 121-125.
14. Sumano López HS, Ocampo Camberos L. 1997. Farmacología Veterinaria, $2^{\circ}$ ed., McGraw-Hill, México, p. 511-513. 